DEPT. OF MATH.

Pure Mathematics

ISSN 0806-2439
UNIV. OF OSLO

No. 12

March 2005

\title{
THE VOLATILITY OF TEMPERATURE AND PRICING OF WEATHER DERIVATIVES
}

\author{
FRED ESPEN BENTH AND JŪRATE் ŠALTYTĖ-BENTH
}

\begin{abstract}
We propose an Ornstein-Uhlenbeck process with seasonal volatility to model the time dynamics of daily average temperatures. The model is fitted to almost 43 years of daily observations recorded in Stockholm, one of the European cities for which there is a trade in weather futures and options on the Chicago Mercantile Exchange (CME). Explicit pricing dynamics for futures contracts written on the number of heating/cooling degree-days (so-called HDD/CDD-futures) and the cumulative average daily temperature (so-called CAT-futures) are calculated, along with a discussion on how to evaluate call and put options with these futures as underlying.
\end{abstract}

\section{INTRODUCTION}

The Chicago Mercantile Exchange (CME) organizes a marketplace for financial derivatives written on temperature. The particpants of the exchange can trade in futures contracts written on temperature indices collected from several US, European as well as two Japanese cities. To obtain reliable prices of the forward products one needs confident stochastic models for the temperature index in question.

The current paper follows up the analysis in Benth and Šaltytė-Benth [3] with a detailed model for the temperature dynamics. As observed in a paper by Campbell and Diebold [6] for US temperature data, and later confirmed for Norwegian temperature data in [3], there is a clear seasonality in the temperature variations after removing yearly mean and meanreversion effects. The seasonality is observed for the autocorrelation function of the squared residuals, which led the authors to search for a seasonality in the volatility of temperature. In this paper we propose a truncated Fourier series to model the seasonal volatility, and validate our model on more than 40 years of daily data collected from Stockholm, Sweden, one of the European cities traded on CME. The volatility model accounts for high variations in the winter periods, together with a higher variation in the summer than spring and fall seasons. Since the form of the autocorrelation function of Stockholm resembles the

Date: March 31, 2005.

Key words and phrases. Weather derivatives, temperature dynamics, stochastic processes, meanreversion, seasonality, Heating degree-day futures, options on temperature.

We are grateful to SMHI, the Swedish Meteorological and Hydrological Institute, for providing us with temperature data. Jūratė Šaltytė-Benth acknowledges the financial support from the Norwegian Research Council, grant NFR: 155120/432. 
observations in [6] for several US cities, we expect our model to perform good also for these.

Our suggested model for daily average temperature variations is a mean-reverting process with seasonality in the level and volatility. It differs from the model of Campbell and Diebold [6] in its simplicity, since they suggest to use an auto-regressive time series model with seasonal ARCH residuals with several lags. This model may be better in fitting to the data (see however, a discussion on this in connection to the Norwegian data in [3]), but we believe that our model is sophisticated enough to explain the basic stylized facts of temperatures, yet simple enough to allow for an explicit derivation of futures prices. Also, for some types of temperature indices, we are able to provide explicit prices for call and put options written on temperature futures typically traded on the CME. We demonstrate the importance of having a realistic model for the seasonality of volatility by looking at HDDfutures prices. Using a constant volatlity for temperature variations leads to significantly lower HDD-futures prices, especially for the summer months where our proposed volatility model gives close to a doubling of the prices.

The mean-reversion model suggested here is a generalization of Doernier and Querel [8]. Even though they state a general Ornstein-Uhlenbeck dynamics with time-dependent variance, they only consider constant specifications in the analysis of Chicago temperature data. Alaton, Djehiche and Stillberger [1] use a similar model for data collected from Bromma, Sweden, with a time-dependent variance. However, they smoothen this out to obtain a constant variance over each month. They calculate prices of several different kinds of temperature derivatives. Finally, we would like to mention the fractional model of Brody, Syroka and Zerovs [5], who consider an Ornstein-Uhlenbeck model with a fractional Brownian motion driving the stochastics. Prices for different temperature derivatives are calculated, however, the derivations become very complicated when going to a timedynamics for different futures products. A different view on pricing weather derivatives is taken by Davis [7], where the HDD-index is modeled directly by a geometric Brownian motion. Options written on this index is priced using marginal value techniques.

In the OTC market, many kinds of temperature derivatives are based on HDD and CDD-futures with different delivery periods than those traded on the CME. Our suggested model for pricing HDD-futures can be used for marking-to-market purposes since the prices are represented as a function of the delivery period. One may first calibrate the model using actual quoted market data from the CME for the contract in question, and next use this to estimate the futures price for the delivery period of interest. In this way we provide a model yielding prices for all possible delivery periods, not only restricted to those on the market place.

The paper is organized as follows. In Section 2 we state our proposed dynamics for the daily temperature variations, and analyse it empirically based on temperature data collected in Stockholm, Sweden, in Section 3. Section 4 is devoted to the derivation of futures prices for different temperature indices like Heating-Degree Days (HDD), CoolingDegree Days (CDD), cumulative average (CAT) and the Asian averaging (Pacific Rim) of temperatures. We also discuss the pricing of options on these derivatives, and include some analytical formulas for average-based futures. The paper concludes in Section 5 . 


\section{Stochastic Dynamics of Temperature VARIATIONS}

Let $(\Omega, \mathcal{F}, P)$ be a complete probability space equipped with a filtration $\left\{\mathcal{F}_{t}\right\}_{t \geq 0}$ satisfying the usual hypotheses (see e.g. Karatzas and Shreve [9]), and denote by $B(t)$ a standard Brownian motion.

We propose the following Ornstein-Uhlenbeck model for the time evolution of temperatures:

$$
d T(t)=d s(t)-\kappa(T(t)-s(t)) d t+\sigma(t) d B(t)
$$

Here, $s(t)$ is a deterministic function modeling the trend and seasonality of temperature, which we assume to be bounded and continuously differentiable. Note that here $T(t)$ is the daily average temperature, usually defined to be the average of the max and min temperature over a $24 \mathrm{~h}$ time span for the date in question. Motivated from the empirical studies in Benth and Saltytè-Benth [2], we suppose that $\sigma(t)$ is a continuously differentiable and bounded function describing the daily volatility of temperature variations. We are in principle free to specify both $s(t)$ and $\sigma(t)$, but as we shall see in the next section, temperature data suggests that choosing a truncated Fourier series for both gives a sufficiently flexible class of functions that at the same time allows for explicit calculations. Thus, from now on we specify $s(t)$ and $\sigma^{2}(t)$ to be of the form

$$
s(t)=a+b t+a_{0}+\sum_{i=1}^{I_{1}} a_{i} \sin \left(2 i \pi\left(t-f_{i}\right) / 365\right)+\sum_{j=1}^{J_{1}} b_{j} \cos \left(2 j \pi\left(t-g_{j}\right) / 365\right),
$$

and

$$
\sigma^{2}(t)=c+\sum_{i=1}^{I_{2}} c_{i} \sin (2 i \pi t / 365)+\sum_{j=1}^{J_{2}} d_{j} \cos (2 j \pi t / 365)
$$

Note that we model the square of $\sigma(t)$ as a truncated Fourier series and not $\sigma(t)$ itself. This is most convenient in the data analysis, but also from the perspective of pricing futures and options since $\sigma^{2}(t)$ is present in many places rather than $\sigma(t)$. Of course, one can use different models than (2.3), however, our experience when analyzing data is that this specification is simple and flexible enough to capture the observed stylized facts of temperatures. In any case, we suppose that the parameters of $\sigma^{2}(t)$ in $(2.3)$ is such that the volatility function is bounded away from zero. We remark in passing that in [2] we used a Lévy process to drive the dynamics when analyzing Norwegian data. In this paper we want to restrict our attention to models where analytical pricing is possible and therefore consider only a Brownian motion driven dynamics. As we shall see for Stockholm, such a model will explain the dynamics to a high degree of accuracy.

Observe that the Itô Formula gives an explicit dynamics of the temperature (2.1):

$$
T(t)=s(t)+(T(0)-s(0)) \mathrm{e}^{-\kappa t}+\int_{0}^{t} \sigma(u) \mathrm{e}^{-\kappa(t-u)} d B(u)
$$


This representation shows us that temperature is normally distributed at each time, but reverting to a mean defined by the seasonal function $s(t)$. The speed of mean-reversion is defined by $\kappa$, and the variance of the temperatures is seasonally varying through $\sigma$.

\section{Analysis of temperature data from Stockholm}

The data we have at hand are average daily temperatures. To fit our stochastic model to these, it is useful to reformulate the time-continuous dynamics to a time series. A straightforward calculation yields

$$
\Delta T(t)=\Delta s(t)-\left(1-\mathrm{e}^{-\kappa}\right)(T(t)-s(t))+\mathrm{e}^{-\kappa} \int_{t}^{t+1} \sigma(u) \mathrm{e}^{-\kappa(t-u)} d B(u),
$$

where the notation $\Delta X(t):=X(t+1)-X(t)$ is used. Approximating the stochastic integral, we find, at least approximately, that

$$
\Delta T(t) \approx \Delta s(t)-\left(1-\mathrm{e}^{-\kappa}\right)(T(t)-s(t))+\mathrm{e}^{-\kappa} \sigma(t) \Delta B(t) .
$$

Thus, when estimating the model (2.1) for the temperature data we consider the following time series model

$$
\widetilde{T}_{t+1}=\alpha \widetilde{T}_{t}+\widetilde{\sigma}(t) \epsilon_{t}
$$

where $\epsilon_{t}$ is i.i.d standard normally distributed, $\alpha=\mathrm{e}^{-\kappa}$, and $\widetilde{\sigma}(t)=\alpha \sigma(t)$.

The fitting procedure will go in several steps. Empirical studies of daily average temperatures show that there is a significant linear trend in the data. Therefore, before we proceed further, we check for the presence of such a trend for the Stockholm temperatures. Next, we fit the seasonal part of $s(t)$ using the method of least squares. Then, we perform a standard linear regression of today's (detrended and deseasonalized) temperatures against previous day's observations. And finally, we estimate the daily variance of the regression residuals, to obtain the $\tilde{\sigma}^{2}(t)$ function. We now describe the results from the estimations in more details.

We have available daily average temperature observations from Stockholm, Sweden, in the period January 1, 1961 untill December 19, 2004, resulting in 16059 records. Data of the last 11 years (starting January 1, 1994) together with the fitted seasonal temperature $s(t)$ are presented in Fig. 1.

A quick look at the histogram (Fig. 2) of the daily average temperatures gives us a clear indication of non-normality of data (indeed, the hypothesis of normality is rejected at the $1 \%$ significance level). The distribution seems to be bimodal, a fact which is not surprising due to the cold and warm seasons in Stockholm. Also we observe a left skewness in data (skewness is equal to -0.10$)$ and negative kurtosis $(-0.66)$.

A simple regression analysis shows that a linear trend exists (in the considered period the daily average temperature increased by approximately $1.6^{\circ} \mathrm{C}$ ) and is significant. The values of intercept and slope are $a=6.28$ and $b=0.0001$, respectively, and they are both significant at the $1 \%$ level.

Using the nlinfit function in MATLAB we fit the seasonal part of the function $s(t)$ to the de-trended data. We set $I_{1}=J_{1}=1$, which leads to the following values of the coefficients: 


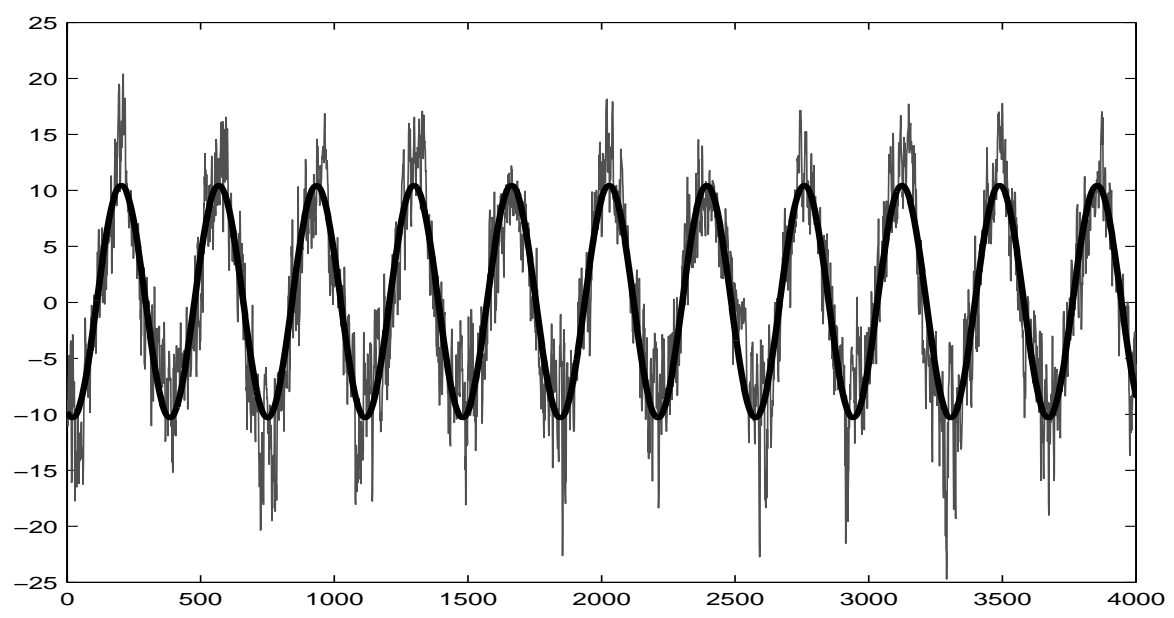

FiguRE 1. Daily average temperatures from Stockholm together with the fitted seasonal function, a snapshot of the last 11 years starting January 1, 1994.

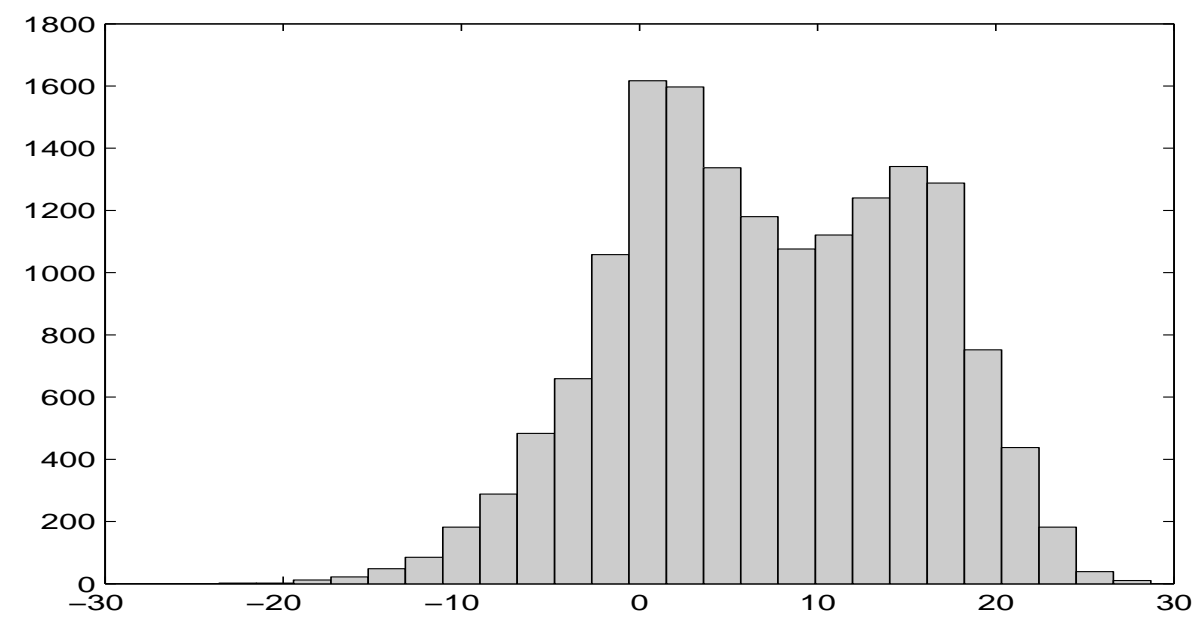

FiguRE 2. Histogram of daily average temperatures from Stockholm.

$a_{0}=0.07, a_{1}=-10.40$ and $b_{1}=22.00$. From Fig. 1 we see that this parametrization is sufficient to capture the seasonal variations in the daily average temperatures.

Next we regress todays de-trended and de-seasonalized daily average temperatures against those of the previous day. In other words, we fit an AR(1)-model which will explain the mean-reversion property of temperature dynamics. The mean-reversion parameter $\alpha=0.82$ is significant at the $1 \%$ level. This estimate of $\alpha$ corresponds to $\kappa=0.198$ in the 
TABLE 1. Fitted parameters of $\sigma^{2}(t)=\widetilde{\sigma}^{2}(t) / \alpha^{2}$

\begin{tabular}{rrrrrrrrr}
\hline$c$ & $c_{1}$ & $c_{2}$ & $c_{3}$ & $c_{4}$ & $d_{1}$ & $d_{2}$ & $d_{3}$ & $d_{4}$ \\
\hline 6.19 & 0.94 & -0.39 & 0.59 & 0.07 & 2.08 & 1.22 & 0.46 & -0.07 \\
\hline
\end{tabular}

original continuous-time dynamics (2.1). Moreover, the constant in the regression analysis was insignificant as expected, and we assume it to be zero. The standard deviation of the residuals is $\bar{\sigma}=2.04$.

The autocorrelation function of the residuals obtained after all effects mentioned above were removed are presented in Fig. 3 (graphics on top). We observe quite high values of the autocorrelation for the several first lags. This indicates that a higher order autoregressive model is called for in a more sophisticated time-series modeling of temperatures. For higher lags, the autocorrelation function seems to vary randomly around zero. However, the autocorrelation function of squared residuals reveals the fact of time dependency in the variance of residuals (see Fig. 3, bottom graphics). Here we see that the first positive lags are rapidly decaying, followed by a clear seasonal variation for larger lags.
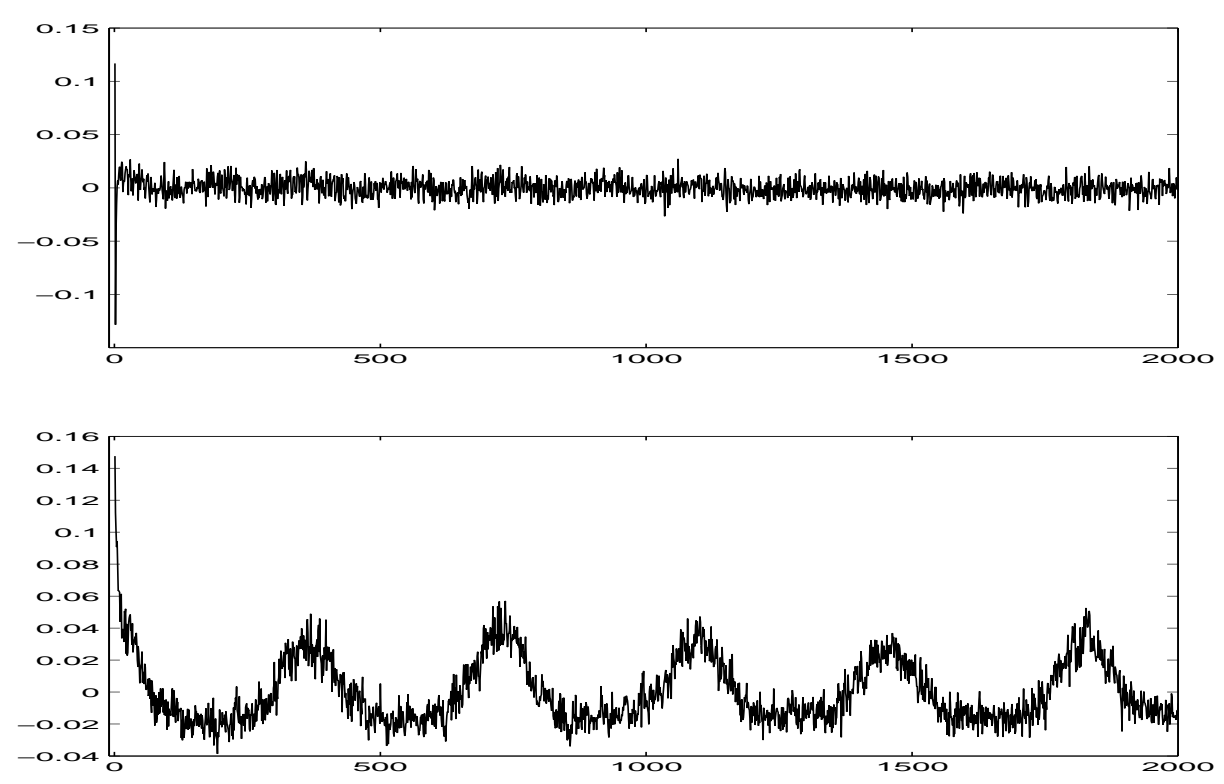

Figure 3. The autocorrelation functions of the residuals and squared residuals of daily average temperatures from Stockholm.

We estimate this temporal dependency in the variance of residuals in the following way. First we find empirical values of the daily variance based on the observations over all years for that particular date. This gives us 365 values. Next we choose $I_{2}=J_{2}=4$ in (2.3), and use nlinfit in MATLAB to estimate the values presented in the Table 1. In Fig. 4 we present the empirical values of $\widetilde{\sigma}^{2}(t)$ together with the fitted function, where we clearly 
observe the temporal dependency in the daily volatility. We have the highest variations in the winter period, while early spring and fall have lower variations than in the summer. Maybe surprisingly, we observe a slight increase in the variance for the summer compared to the autumn and fall seasons, with the lowest variation in the fall.

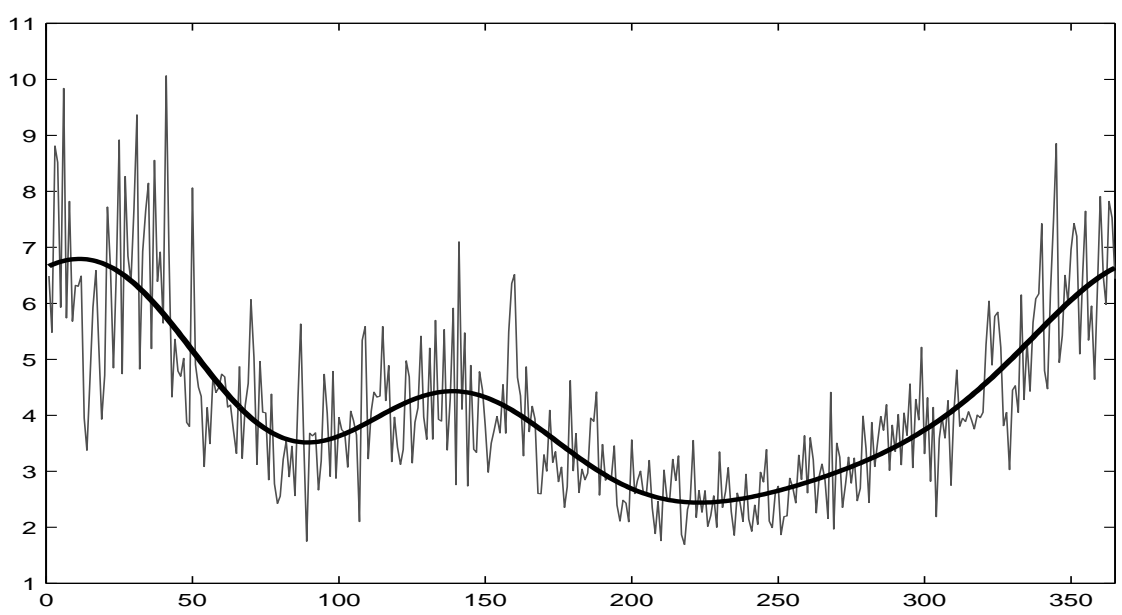

FIGURE 4. Empirical daily squared volatility together with the fitted volatility function $\widetilde{\sigma}^{2}(t)$

The residuals and squared residuals obtained after removing the temporal phenomena in the variance are presented in Fig. 5. As we can see, we have completely removed the seasonality in the autocorrelation function for squared residuals. The rapid decay in the autocorrelation for the first lags is still present, suggesting that a more refined model could be of GARCH-type (see e.g. Bollerslev [4]). However, this will complicate the calculation of futures and options prices significantly, and we will not consider such an extension in this paper.

The histogram of the residuals (Fig. 6) seems to be quite unimodal and symmetrical. We find the skewness to be -0.010 and a kurtosis equal to 0.004 . However, the hypothesis of normality is rejected at the $1 \%$ significance level. The same conclusion was made for the Norwegian temperature data analyzed in [3], and there we suggested to model the residuals by a generalized hyperbolic distribution. This family of distributions are able to capture the small peak in the center and the semi-heavy tails that we found present in the Norwegian data. A GARCH-model for the residuals may produce similar results. On the other hand, these effects are small. The inclusion of a non-normal model for the residuals leads to a complicated Lévy process dynamics which may be hard to use for pricing of derivatives. We believe that the assumption about i.i.d standard normal residuals is a reasonable one.

We now move on to calculate futures and option prices based on our model, where we will provide some numerical examples using the estimated model of Stockholm temperatures. 

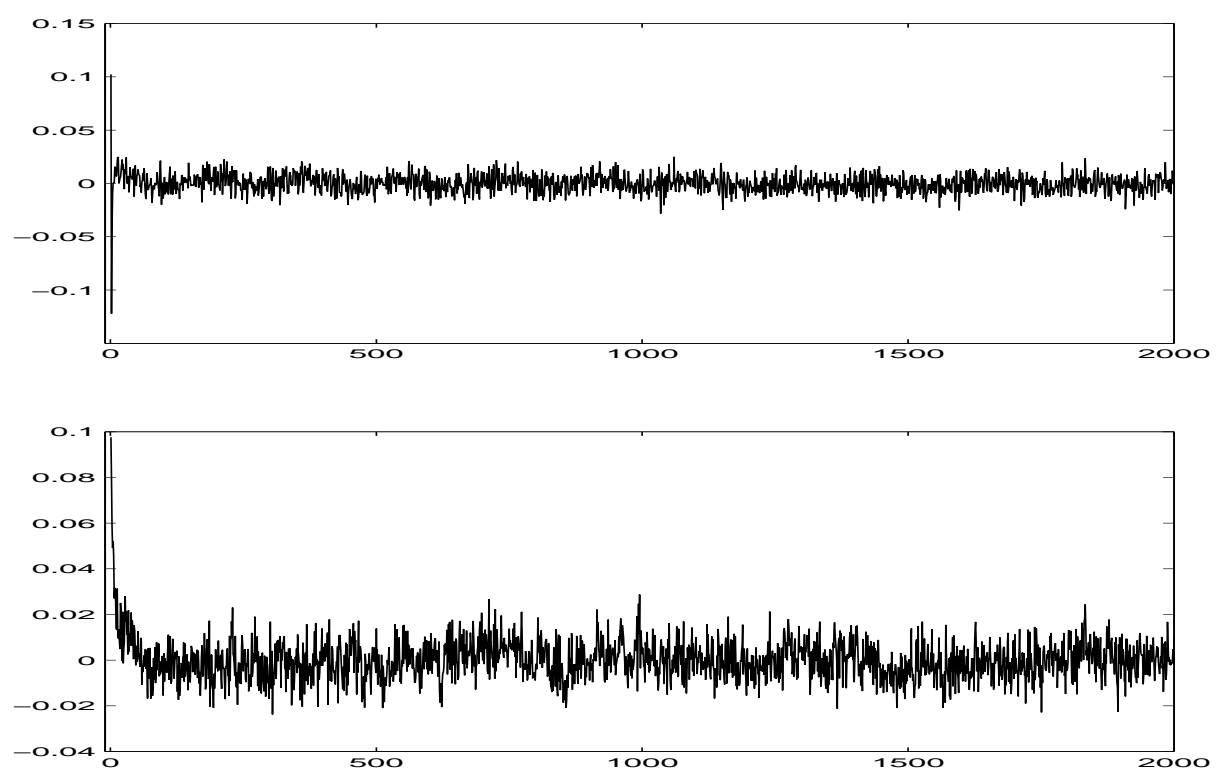

FiguRE 5. Autocorrelation function for squared residuals before (above) and after (below) dividing out the volatility function $\widetilde{\sigma}(t)$ from the regression residuals.

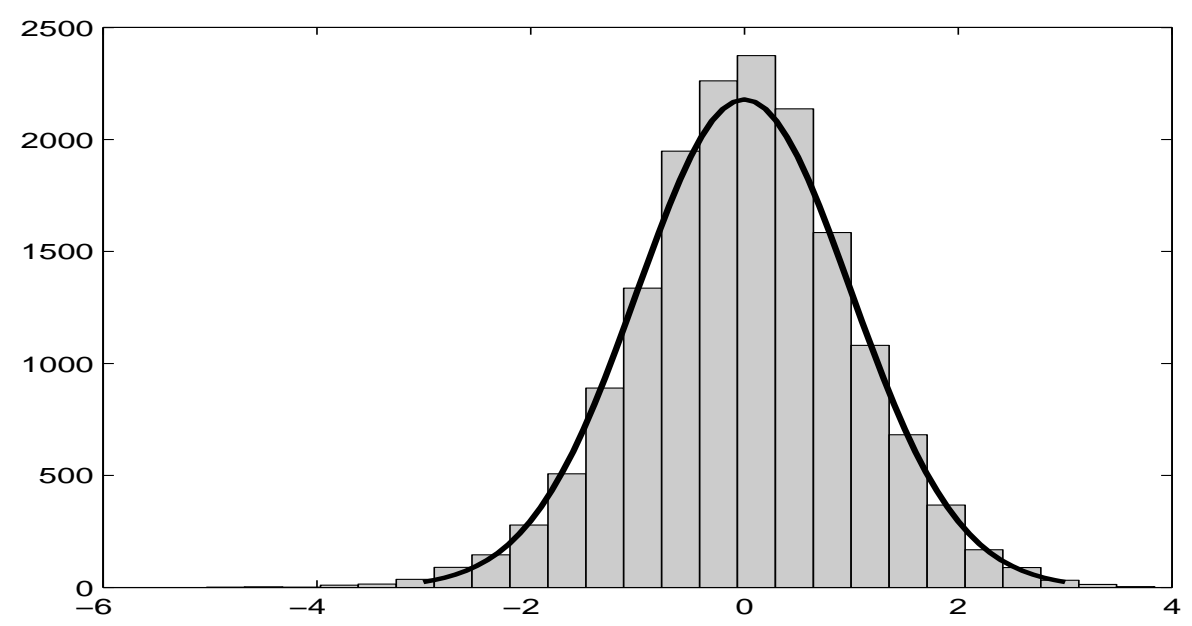

FiguRE 6. Histogram of the residuals of temperatures from Stockholm together with the standard normal density.

\section{Derivatives on Temperature}

The Chicago Mercantile Exchange (CME) organizes standardized trading in futures and options written on temperature indices for several US, European and Japanese cities ${ }^{1}$.

\footnotetext{
${ }^{1}$ See $h t t p: / / w w w . c m e . c o m / p r d / w e c /$ for more information about this trading.
} 
The futures have the number of Heating-Degree Days (HDD) (or Cooling-Degree days (CDD)) over one month or one season for 15 US cities as the underlying temperature index. The HDD index over the time interval $\left[\tau_{1}, \tau_{2}\right]$ is defined in a continuous-time setting as $\int_{\tau_{1}}^{\tau_{2}} \max (65-T(\tau), 0) d \tau$, whereas the CDD index is defined as $\int_{\tau_{1}}^{\tau_{2}} \max (T(\tau)-65,0) d \tau$. The name CDD is stemming from the fact that most air-conditioning is switched on when temperatures goes above $65^{\circ} \mathrm{F}$, while heating is mostly used for lower temperatures, explaining the name HDD. The futures written on these two indices are financially settled at the end of the index period.

For the 5 European cities one can trade in futures written on the cumulative (average) temperature (CAT) index and the HDD index over a month or season. The CAT index over a time interval $\left[\tau_{1}, \tau_{2}\right]$ is defined as $\int_{\tau_{1}}^{\tau_{2}} T(\tau) d \tau$, where the temperature is measured in degrees of Celsius and not Fahrenheit. The contracts are denominated in GBP rather than USD. Furthermore, the temperature level for the HDD-contracts is set to $18^{\circ} \mathrm{C}$.

For the two Japanese cities (Tokyo and Osaka), the futures are written on the so-called Pacific Rim index, which measures the average daily temperature over a month or season. The Pacific Rim index over the period $\left[\tau_{1}, \tau_{2}\right]$ is defined as $\int_{\tau_{1}}^{\tau_{2}} T(\tau) d \tau /\left(\tau_{2}-\tau_{1}\right)$, and the contracts are denominated in Japanese Yen.

The CME also organizes a trade in plain vanilla European options on the different temperature index futures. For all HDD, CDD, CAT and Pacific Rim index futures there exist call and put options for different strikes and maturities.

Let us consider the price dynamics of futures written on the HDD index over a specified period $\left[\tau_{1}, \tau_{2}\right], \tau_{1}<\tau_{2}$ (the winter season, say). Assuming a constant continuously compounding interest rate $r$, the futures price at time $t \leq \tau_{1}$ written on the HDD index is defined as the $\mathcal{F}_{t}$-adapted stochastic process $F_{\mathrm{HDD}}\left(t, \tau_{1}, \tau_{2}\right)$ satisfying $^{2}$

$$
0=\mathrm{e}^{-r\left(\tau_{2}-t\right)} \mathbb{E}_{Q}\left[\int_{\tau_{1}}^{\tau_{2}} \max (c-T(\tau), 0) d \tau-F_{\mathrm{HDD}}\left(t, \tau_{1}, \tau_{2}\right) \mid \mathcal{F}_{t}\right] .
$$

Here $Q$ is a risk-neutral probability and $c$ is equal to $65^{\circ} \mathrm{F}$ or $18^{\circ} \mathrm{C}$ depending on whether the contract is for a US or European city. From the adaptedness of $F_{\mathrm{HDD}}\left(t, \tau_{1}, \tau_{2}\right)$, we easily find the futures price to be

$$
F_{\mathrm{HDD}}\left(t, \tau_{1}, \tau_{2}\right)=\mathbb{E}_{Q}\left[\int_{\tau_{1}}^{\tau_{2}} \max (c-T(\tau), 0) d \tau \mid \mathcal{F}_{t}\right],
$$

Analogously, the CDD-futures price is

$$
F_{\mathrm{CDD}}\left(t, \tau_{1}, \tau_{2}\right)=\mathbb{E}_{Q}\left[\int_{\tau_{1}}^{\tau_{2}} \max (T(\tau)-c, 0) d \tau \mid \mathcal{F}_{t}\right],
$$

By the same reasoning, we derive the price of a CAT-futures and a Pacific Rim-futures to be

$$
F_{\mathrm{CAT}}\left(t, \tau_{1}, \tau_{2}\right)=\mathbb{E}_{Q}\left[\int_{\tau_{1}}^{\tau_{2}} T(\tau) d \tau \mid \mathcal{F}_{t}\right]
$$

\footnotetext{
${ }^{2}$ We have assumed that settlement of the contract takes place at the end of the period where the index is created, namely $\tau_{2}$.
} 
and

$$
F_{\mathrm{PRIM}}\left(t, \tau_{1}, \tau_{2}\right)=\mathbb{E}_{Q}\left[\frac{1}{\tau_{2}-\tau_{1}} \int_{\tau_{1}}^{\tau_{2}} T(\tau) d \tau \mid \mathcal{F}_{t}\right]
$$

Observe that trivially,

$$
F_{\mathrm{PRIM}}\left(t, \tau_{1}, \tau_{2}\right)=\frac{1}{\tau_{2}-\tau_{1}} F_{\mathrm{CAT}}\left(t, \tau_{1}, \tau_{2}\right)
$$

Furthermore, since $\max (c-x, 0)=c-x+\max (x-c, 0)$, we have

$$
F_{\mathrm{HDD}}\left(t, \tau_{1}, \tau_{2}\right)=c\left(\tau_{2}-\tau_{1}\right)-F_{\mathrm{CAT}}\left(t, \tau_{1}, \tau_{2}\right)+F_{\mathrm{CDD}}\left(t, \tau_{1}, \tau_{2}\right) .
$$

In order to derive a more explicit expression for the futures price, we need to specify the risk-neutral probability $Q$. Since temperature is not a storable commodity, the futures contracts can not be hedged and the market is therefore incomplete. A risk-neutral probability is by definition a probability measure $Q \sim P$ such that all tradeable assets in the market are martingales after discounting. Thus, all equivalent probabilities $Q$ will become risk-neutral probabilities. We specify a sub-family of probability measures $Q$ using the Girsanov transform: Assume $\theta(t)$ is a real-valued measurable and bounded function. The stochastic process

$$
\left.Z^{\theta}(t)=\exp \left(\int_{0}^{t} \frac{\theta(s)}{\sigma(s)} d B(s)-\frac{1}{2} \int_{0}^{t} \frac{\theta^{2}(s)}{\sigma^{2}(s)}\right) d s\right)
$$

will become the density process of the probability measure

$$
Q^{\theta}(A)=\mathbb{E}\left[1_{A} Z^{\theta}\left(\tau_{\max }\right)\right],
$$

where $1_{A}$ is the indicator function and $\tau_{\max }$ is a fixed time horizon bigger than the trading times for all relevant futures. This probability is obviously equivalent to $P$, and the process

$$
d W(t)=d B(t)-\frac{\theta(t)}{\sigma(t)} d t
$$

is a standard Brownian motion under $Q^{\theta}$. Note that we assumed that the parameters of $\sigma^{2}(t)$ are such that the function is bounded away from zero, thus creating no problems when using it as a divisor in the Girsanov transformation. The temperature dynamics under $Q^{\theta}$ becomes

$$
d T(t)=d s(t)+(\theta(t)-\kappa(T(t)-s(t))) d t+\sigma(t) d W(t) .
$$

We denote the expectation under the probability $Q^{\theta}$ by $\mathbb{E}_{\theta}[\cdot]$. By using time-varying $\theta^{\prime}$ s we have a flexible class of risk-neutral probabilities $Q^{\theta}$ that we easily can fit to the observed forward curves. We call $\theta$ the "market price of risk". An explicit form of $T(t)$ is given by

$$
T(t)=s(t)+(T(0)-s(0)) \mathrm{e}^{-\kappa t}+\int_{0}^{t} \theta(u) \mathrm{e}^{-\kappa(t-u)} d u+\int_{0}^{t} \sigma(u) \mathrm{e}^{-\kappa(t-u)} d W(u) .
$$

We see that $T(t)$ is normally distributed under $Q^{\theta}$, with expectation

$$
\mathbb{E}_{\theta}[T(t)]=s(t)(T(0)-s(0)) \mathrm{e}^{-\kappa t}+\int_{0}^{t} \theta(u) \mathrm{e}^{-\kappa(t-u)} d u
$$


and variance

$$
\operatorname{Var}_{\theta}[T(t)]=\int_{0}^{t} \sigma^{2}(u) \mathrm{e}^{-2 \kappa(t-u)} d u
$$

We now move on to calculate the futures prices for the contracts traded at the CME.

4.1. HDD/CDD-futures and options. Denote by $\Phi$ the cumulative probability distribution function of a standard normal variable, and $\phi$ its density. We have the following result for the price of a HDD-futures.

Proposition 4.1. The price of a HDD-futures at time $t$, where the index is measured over the period $\left[\tau_{1}, \tau_{2}\right], t \leq \tau_{1}<\tau_{2}$, is given by

$$
F_{H D D}\left(t, \tau_{1}, \tau_{2}\right)=\int_{\tau_{1}}^{\tau_{2}} \Sigma(t, \tau)\{d(t, \tau, T(t)) \Phi(d(t, \tau, T(t)))+\phi(d(t, \tau, T(t)))\} d \tau
$$

where

$$
\begin{aligned}
d(t, \tau, x) & =\frac{c-s(\tau)+(s(t)-x) e^{-\kappa(\tau-t)}-\int_{t}^{\tau} \theta(u) e^{-\kappa(\tau-u)} d u}{\Sigma(t, \tau)} \\
\Sigma^{2}(t, \tau) & =\int_{t}^{\tau} \sigma^{2}(u) e^{-2 \kappa(\tau-u)} d u .
\end{aligned}
$$

Proof. By the Fubini-Tonelli theorem, we can interchange expectation and integration to obtain

$$
F_{\mathrm{HDD}}\left(t, \tau_{1}, \tau_{2}\right)=\int_{\tau_{1}}^{\tau_{2}} \mathbb{E}_{\theta}\left[\max (c-T(\tau), 0) \mid \mathcal{F}_{t}\right] d \tau
$$

We now calculate

$$
\mathbb{E}_{\theta}\left[\max (c-T(\tau), 0) \mid \mathcal{F}_{t}\right]
$$

by using Fourier transform techniques. To make notation more compact, let $f(x)=$ $\max (c-x)$. Observe that $f$ is not an integrable function over $\mathbb{R}$, and we dampen it with an exponential function $\mathrm{e}^{\alpha x}$ with $\alpha>0$ and define

$$
f_{\alpha}(x)=\mathrm{e}^{\alpha x} f(x) \text {. }
$$

It is easily seen that $f_{\alpha} \in L^{1}(\mathbb{R})$. From Fourier analysis we know that

$$
\mathrm{e}^{\alpha x} f(x)=\frac{1}{2 \pi} \int \widehat{f}_{\alpha}(y) \mathrm{e}^{\mathrm{i} x y} d y
$$

and thus

$$
\mathbb{E}_{\theta}\left[\max (c-T(\tau), 0) \mid \mathcal{F}_{t}\right]=\frac{1}{2 \pi} \int \widehat{f}_{\alpha}(y) \mathbb{E}_{\theta}\left[\mathrm{e}^{(-\alpha+\mathrm{i} y) T(\tau)} \mid \mathcal{F}_{t}\right] d y .
$$

Let $z:=-\alpha+\mathrm{i} y$. Then

$$
\mathbb{E}_{\theta}\left[\mathrm{e}^{z T(\tau)} \mid \mathcal{F}_{t}\right]=\exp \left(z s(\tau)+z(T(0)-s(0)) \mathrm{e}^{-\kappa \tau}+z \int_{0}^{\tau} \theta(u) \mathrm{e}^{-\kappa(\tau-u)} d u\right)
$$




$$
\begin{aligned}
& \times \mathbb{E}_{\theta}\left[\exp \left(z \int_{0}^{\tau} \sigma(u) \mathrm{e}^{-\kappa(\tau-u)} d W(u)\right) \mid \mathcal{F}_{t}\right] \\
& =\exp \left(z s(\tau)+z(T(0)-s(0)) \mathrm{e}^{-\kappa \tau}+z \int_{0}^{\tau} \theta(u) \mathrm{e}^{-\kappa(\tau-u)} d u\right. \\
& \left.\quad+z \int_{0}^{t} \sigma(u) \mathrm{e}^{-\kappa(\tau-u)} d W(u)\right) \mathbb{E}_{\theta}\left[\exp \left(z \int_{t}^{\tau} \sigma(u) \mathrm{e}^{-\kappa(\tau-u)} d W(u)\right)\right] \\
& =\exp \left(z s(\tau)+z(T(t)-s(t)) \mathrm{e}^{-\kappa(\tau-t)}+z \int_{t}^{\tau} \theta(u) \mathrm{e}^{-\kappa(\tau-u)} d u\right) \\
& \quad \times \exp \left(\frac{1}{2} z^{2} \int_{t}^{\tau} \sigma^{2}(u) \mathrm{e}^{-2 \kappa(\tau-u)} d u\right)
\end{aligned}
$$

where we used the measurability and independent increment property of the Wiener process in the second to the last equality.

Introduce the notation

$$
c(t, \tau)=s(\tau)+(T(t)-s(t)) \mathrm{e}^{-\kappa(\tau-t)}+\int_{t}^{\tau} \theta(u) \mathrm{e}^{-\kappa(\tau-u)} d u .
$$

Thus,

$$
\begin{aligned}
\mathbb{E}_{\theta}\left[f(T(t)) \mid \mathcal{F}_{t}\right] & =\frac{1}{2 \pi} \int \widehat{f}_{\alpha}(y) \mathrm{e}^{-\frac{1}{2} y^{2} \Sigma^{2}(t, \tau)} \mathrm{e}^{\mathrm{i} y c(t, \tau)-\mathrm{i} y \alpha \Sigma^{2}(t, \tau)} d y \mathrm{e}^{\frac{1}{2} \alpha^{2} \Sigma^{2}(t, \tau)-\alpha c(t, \tau)} \\
& =\mathrm{e}^{\frac{1}{2} \alpha^{2} \Sigma^{2}(t, \tau)-\alpha c(t, \tau)}\left(f_{\alpha} \star p\right)\left(c(t, \tau)-\alpha \Sigma^{2}(t, \tau)\right)
\end{aligned}
$$

where $p(x)$ is the density function of a zero-mean normal random variable with variance $\Sigma^{2}(t, \tau)$. Appealing to the definition of $f_{\alpha}$, we find

$$
\mathbb{E}_{\theta}\left[f(T(t)) \mid \mathcal{F}_{t}\right]=\mathbb{E}\left[\mathrm{e}^{X \alpha \Sigma(t, \tau)} \max \left(\left(c-c(t, \tau)+\alpha \Sigma^{2}(t, \tau)\right)-X \Sigma(t, \tau), 0\right)\right],
$$

for $X$ being a standard normal random variable. A straightforward calculation of this expectation yields the result.

By appealing to the Itô Formula, we can derive the dynamics of the HDD-futures price, $d F_{\mathrm{HDD}}\left(t, \tau_{1}, \tau_{2}\right)$.

Proposition 4.2. The time-dynamics of a HDD-futures where the index is measured over the period $\left[\tau_{1}, \tau_{2}\right]$ is given by

$$
d F_{H D D}\left(t, \tau_{1}, \tau_{2}\right)=-\sigma(t) \int_{\tau_{1}}^{\tau_{2}} e^{-\kappa(\tau-t)} \Phi(d(t, \tau, T(t))) d \tau d W(t)
$$

for $t \leq \tau_{1}<\tau_{2}$. The function $d(t, \tau, T(t))$ is defined in Prop. 4.1 above.

Proof. Recall the notation in Prop. 4.1 and its proof. We know that $F_{\mathrm{HDD}}\left(t, \tau_{1}, \tau_{2}\right)$ is a $Q^{\theta}$-martingale, and therefore we need only to focus on the " $d W$ "-term in Itô's Formula:

$d F_{\mathrm{HDD}}\left(t, \tau_{1}, \tau_{2}\right)=\int_{\tau_{1}}^{\tau_{2}}\left(-\mathrm{e}^{-\kappa(\tau-t)} \Phi(d(t, \tau, T(t)))-\left(c(t, \tau)-T(t) \mathrm{e}^{-\kappa(\tau-t)}\right)\right.$ 


$$
\begin{aligned}
& \left.\times \phi(d(t, \tau, T(t))) \frac{\mathrm{e}^{-\kappa(\tau-t)}}{\Sigma(t, \tau)}+\phi(d(t, \tau, T(t))) \mathrm{e}^{-\kappa(\tau-t)} d(t, \tau, T(t))\right) d \tau \sigma(t) d W(t) \\
= & -\int_{\tau_{1}}^{\tau_{2}} \sigma(t) \mathrm{e}^{-\kappa(\tau-t)} \Phi(d(t, \tau, T(t))) d \tau d W(t) .
\end{aligned}
$$

This concludes the proof.

The dynamics of $F_{\mathrm{HDD}}$ is rather complicated and does not lend itself to explicit computations of put and call options prices, something which is confirmed by the explicit expression in (4.12). Thus, we are left with a numerical approach if we want to find prices of calls and puts written on HDD- (or CDD-) futures. A simple approach could be a Monte Carlo method simulating the risk-neutral temperature $T(\tau)$ at the strike time $\tau$, and then performing a numerical integration to derive $F_{\mathrm{HDD}}\left(\tau, \tau_{1}, \tau_{2}\right)$.

Note that we need a specification of the market price of risk $\theta$ in order to find the HDD-futures price, and options prices written on these futures. By choosing an appropriate family of functions $\theta$, we can fit these using today's observed HDD-futures curve by appealing to the theoretical price curve yielded by (4.12):

$$
F_{\mathrm{HDD}}\left(0, \tau_{1}, \tau_{2}\right)=\int_{\tau_{1}}^{\tau_{2}} \Sigma(0, \tau)\{d(0, \tau, T(0)) \Phi(d(0, \tau, T(0)))+\phi(d(0, \tau, T(0)))\} d \tau
$$

The dependency on $\theta$ is in the function $d$ (see Prop. 4.1). In Fig. 7 we have plotted the HDD-futures curve (4.14) at time 0 for each month the following year based on the fitted temperature model of Stockholm reported in the previous section. We have assumed zero market price of risk, i.e. $\theta=0$. In the same plot we have included the HDD-futures curve we would obtain if $\sigma(t)$ is supposed to be a constant (broken lines). The constant is chosen to be the standard deviation of the residuals from the regression analysis, which we recall to be $\bar{\sigma}=2.04$. We observe that assuming a constant volatility leads to an underestimating of the futures curve, consequently for each month through the year. In the winter the error is small, but in the summer we see that the varying volatility accounts for close to a doubling of the price. This emphasizes the importance of having a good model for the temperature volatility when pricing futures contracts.

4.2. CAT and Pacific Rim-futures and options. In Benth and Šaltytè-Benth [2] we calculated explicitly the CAT-futures price dynamics based on the model (2.1) with Lévy dynamics. We include here the results and proofs in the special case of a Brownian motion for the sake of completeness.

First let us calculate the cumulative temperature over a time interval $\left[\tau_{1}, \tau_{2}\right]$ under the risk neutral probability $Q^{\theta}$ :

Lemma 4.3. If the temperature $T(t)$ follows (4.10), the cumulative temperature over the time interval $\left[\tau_{1}, \tau_{2}\right]$ is explicitly given by

$$
\int_{\tau_{1}}^{\tau_{2}} T(t) d t=\int_{\tau_{1}}^{\tau_{2}} s(t) d t-\kappa^{-1}(T(0)-s(0))\left(e^{-\kappa \tau_{2}}-e^{-\kappa \tau_{1}}\right)
$$




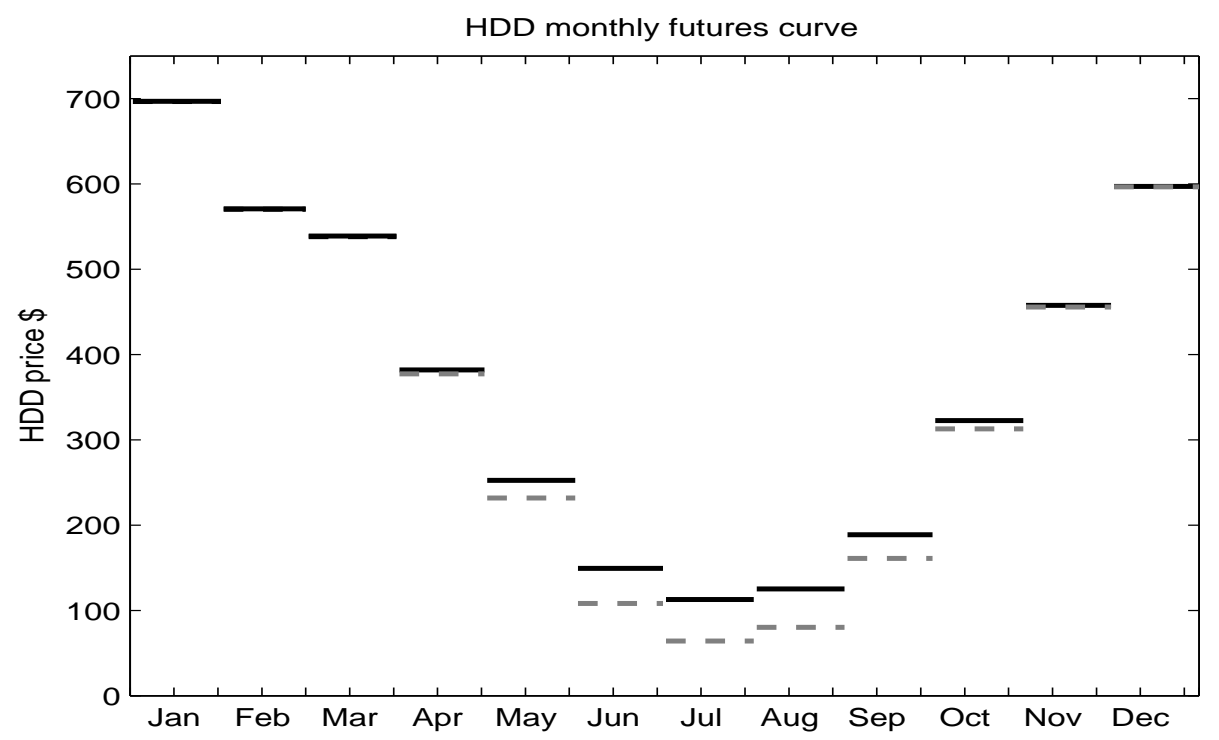

Figure 7. The HDD-futures curve (4.14) for each month based on the model for Stockholm temperatures. The broken lines represent the the corresponding HDD-prices when assuming a constant volatility of temperature $\bar{\sigma}=2.04$.

$$
\begin{aligned}
& -\int_{0}^{\tau_{2}} \theta(t) \kappa^{-1}\left\{e^{-\kappa\left(\tau_{2}-t\right)}-\mathbf{1}_{\left[0, \tau_{1}\right]}(t) e^{-\kappa\left(\tau_{1}-t\right)}-\mathbf{1}_{\left[\tau_{1}, \tau_{2}\right]}(t)\right\} d t \\
& -\int_{0}^{\tau_{2}} \sigma(t) \kappa^{-1}\left\{e^{-\kappa\left(\tau_{2}-t\right)}-\mathbf{1}_{\left[0, \tau_{1}\right]}(t) e^{-\kappa\left(\tau_{1}-t\right)}-\mathbf{1}_{\left[\tau_{1}, \tau_{2}\right]}(t)\right\} d W(t) .
\end{aligned}
$$

Proof. Let $\widetilde{T}(t)=T(t)-s(t)$ be the deseasonalized temperature. From (4.10) we find

$$
\widetilde{T}\left(\tau_{2}\right)=\widetilde{T}\left(\tau_{1}\right)-\kappa \int_{\tau_{1}}^{\tau_{2}} \widetilde{T}(t) d t+\int_{\tau_{1}}^{\tau_{2}} \theta(t) d t+\int_{\tau_{1}}^{\tau_{2}} \sigma(t) d W(t)
$$

Combining this with the explicit dynamics of $T(t)$ in (4.11) yield the Lemma.

We apply this result to calculate the CAT-futures price.

Proposition 4.4. The CAT-futures price $F_{C A T}\left(t, \tau_{1}, \tau_{2}\right)$ at time $t \leq \tau_{1}$ where the index is measured over the interval $\left[\tau_{1}, \tau_{2}\right]$ is given by,

$$
F_{C A T}\left(t, \tau_{1}, \tau_{2}\right)=\int_{\tau_{1}}^{\tau_{2}} s(\tau) d \tau+\kappa^{-1}\left(e^{-\kappa\left(\tau_{1}-t\right)}-e^{-\kappa\left(\tau_{2}-t\right)}\right)(T(t)-s(t))+\Theta\left(t, \tau_{1}, \tau_{2}\right)
$$

where $\Theta\left(t, \tau_{1}, \tau_{2}\right)$ is given as a function of the market price of risk and volatility as

$$
\Theta\left(t, \tau_{1}, \tau_{2}\right)=\kappa^{-1} \int_{t}^{\tau_{2}} \theta(u)\left(1-e^{-\kappa\left(\tau_{2}-u\right)}\right) d u
$$




$$
-\kappa^{-1} \int_{t}^{\tau_{1}} \theta(u)\left(1-e^{-\kappa\left(\tau_{1}-u\right)}\right) d u .
$$

Proof. This is a simple calculation using (4.11), (4.15) together with the independent increment property of Brownian motion.

A straightforward application of Itô's Formula gives

$$
d F_{\mathrm{CAT}}\left(t, \tau_{1}, \tau_{2}\right)=\Sigma\left(t, \tau_{1}, \tau_{2}\right) d W(t) .
$$

where

$$
\Sigma\left(t, \tau_{1}, \tau_{2}\right):=\kappa^{-1}\left(\mathrm{e}^{-\kappa\left(\tau_{1}-t\right)}-\mathrm{e}^{-\kappa\left(\tau_{2}-t\right)}\right) \sigma(t) .
$$

We can interpret $\Sigma\left(t, \tau_{1}, \tau_{2}\right)$ as the volatility term structure of the CAT-futures dynamics. In Fig. 8 we have plotted the volatility as a function of time untill the beginning of each delivery month. The first curve is $\Sigma\left(t, \tau_{1}, \tau_{2}\right)$ plotted for $t$ starting January 1 , and ending January 31, and delivery is in February. Next curve is the volatility for delivery in March, with time ranging from January 1 untill end of February, and so on untill delivery in January next year. The parameters are taken from the Stockholm model. We notice that there is hardly any variation before we are close to delivery, where the volatility is sharply incrasing. This is in line with observations in commodity markets, where there is a maturity effect in the volatility. Samuelson [10] argued for an increasing volatility of futures price returns as time to maturity decreases, since most of the important information is revealed close to maturity. Our model predicts a similar behaviour for the variation of the absolute CAT-futures prices. The seasonality in $\sigma(t)$ is apparent from the different

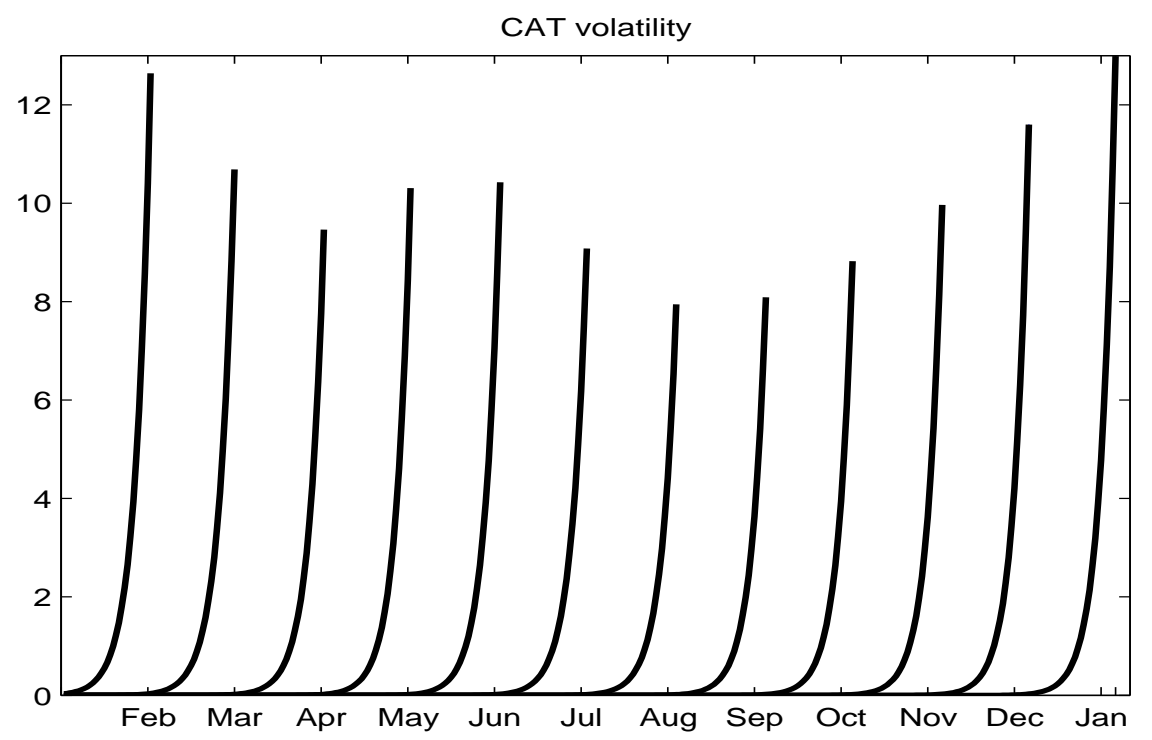

FiguRE 8 . The volatility $\Sigma\left(t, \tau_{1}, \tau_{2}\right)$ for every month ranging from February to January. Time is running from January 1 up to the day before delivery. 
levels of volatility close to maturity. Also we observe that there is hardly any volatility far from the delivery period, since in view of (4.16) the stochastic component $T(t)-s(t)$ of the CAT-futures price is negligible.

Since the CAT-futures price is an additive Gaussian process, we can easily derive an explicit formula for a call option written on the futures contract having exercise date $\tau \leq \tau_{1}$ and strike price $K$.

Proposition 4.5. The price of a call option at time $t$ on the CAT-futures contract with exercise date $t \leq \tau \leq \tau_{1}$ and strike price $K$ is

$$
C(t)=e^{-r(\tau-t)}\left(F_{C A T}\left(t, \tau_{1}, \tau_{2}\right)-K\right) \Phi(d)+\frac{\Sigma_{t, \tau}}{\sqrt{2 \pi}} e^{-d^{2} / 2},
$$

where

$$
d=\frac{F_{C A T}\left(t, \tau_{1}, \tau_{2}\right)-K}{\Sigma_{t, \tau}}, \quad \Sigma_{t, \tau}^{2}:=\int_{t}^{\tau} \Sigma^{2}\left(u, \tau_{1}, \tau_{2}\right) d u
$$

$\Phi$ the cumulative probability function for the standard normal distribution, and $\Sigma\left(t, \tau_{1}, \tau_{2}\right)$ is defined in (4.19).

Proof. This is a straightforward calculation using the properties of the normal distribution.

By simple modifications of the analysis above we can derive explicitly the price of PRIMfutures and call options written on these, as well.

\section{Conclusions}

We have proposed a simple model for the time-dynamics of daily average temperatures. The model is a mean-reverting stochastic process, which has a seasonal variance function explaining the seasonality observed in the temperature residuals. Our model is simple, but yet powerful enough to describe the most apparent stylized facts of temperature data like seasonality and mean-reversion. The seasonal variance function is parametrized as a truncated Fourier series, and we fit the model to more than 40 years of daily data collected from Stockhom, Sweden.

The simplicity of our proposed dynamics allows for explicit calculation of futures prices for HDD/CDD, CAT and PRIM futures quoted on the Chicago Mercantile Exchange. We also provide explicit formulas for options written on CAT/PRIM futures. The expressions include a time-dependent market price of risk function, which opens for a seasonal modeling of this.

We demonstrate empirically that the HDD-futures curve gives higher prices when taking into account a seasonal volatility of temperature compared to a constant volatility. The difference is significant in the summer period, where we experience close to a doubling of prices with a time-dependent volatility. We also show that there is a seasonal maturity effect in the volatility of CAT-futures prices. 


\section{REFERENCES}

[1] Alaton, P., Djehiche, B. and Stillberger, D. (2002). On modelling and pricing weather derivatives. Appl. Math. Finance, 9(1), 1-20.

[2] Benth, F. E. and Saltyte-Benth, J. (2004). The normal inverse Gaussian distribution and spot price modelling in energy markets. Intern. J. Theor. Appl. Finance, 7(2), 177-192.

[3] Benth, F. E. and Šaltytė-Benth, J. (2004). Stochastic modelling of temperature variations with a view towards weather derivatives. To appear in Appl. Math. Finance.

[4] Bollerslev, T. (1986). Generalized autoregressive conditional heteroscedasticity. J. Econometrics, 31, 307-327.

[5] Brody, D. C., Syroka, J. and Zervos, M. (2002). Dynamical pricing of weather derivatives. Quantit. Finance, 3, 189-198.

[6] Campbell, S. D. and Diebold, F. X. (2002). Weather forecasting for weather derivatives. Manuscript. Available on http://www.ssc.upenn.edu/ fdiebold/papers/papers.html. Version: December 4, 2002.

[7] Davis, M. H. A. (2001). Pricing weather derivatives by marginal value. Quantit. Finance, 1, 305-308.

[8] Dornier, F. and Querel, M. (2000). Caution to the wind. Energy Power Risk Manag., Weather risk special report, August, 30-32.

[9] Karatzas, I., and Shreve, S. E. (1991). Brownian Motion and Stochastic Calculus, Springer-Verlag, New York.

[10] Samuelson, P. (1965). Rational theory of warrant pricing. Indust. Management Rev., 6, pp. 13-32.

(Fred Espen Benth)

Centre of Mathematics for Applications

UNIVERSITY OF OSLO

P.O. Box 1053, BLINDERN

N-0316 Oslo, Norway

AND

Agder University College

Department of Economics and Business Administration

SERVICEBOKS 422

N-4604 Kristiansand, Norway

E-mail address: fredb@math.uio.no

URL: http://www.math.uio.no/ fredb/

(Jūratè Šaltytè-Benth)

Centre of Mathematics for Applications

UNIVERSITY OF OSLO

P.O. Box 1053, BLINDERN

N-0316 OsLo, Norway

AND

Department of System Research

KLAIPĖDA UNIVERSITY

H. MANTO 84

LT-5808 KLAIPE்DA, LiTHUANIA

E-mail address: jurate@math.uio.no 